\title{
Inhibitory effect of norcantharidin on melanoma tumor growth and vasculogenic mimicry by suppressing MMP-2 expression
}

\author{
ZHENYU WANG ${ }^{1}$, DINGYUN YOU ${ }^{2}$, MINNAN LU $^{3}$, YUEFENG HE $^{4}$ and SHAN YAN ${ }^{5}$ \\ ${ }^{1}$ Biomedical Engineering Research Center, Kunming Medical University; ${ }^{2}$ Department of Science and Technology; \\ ${ }^{3}$ Experimental Center for Medical Science Research; ${ }^{4}$ School of Public Health; ${ }^{5}$ Institute of Molecular and Clinical Medicine, \\ Kunming Medical University, Yunnan, Kunming 650500, P.R. China
}

Received April 29, 2015; Accepted September 9, 2016

DOI: $10.3892 / \mathrm{ol} .2017 .5622$

\begin{abstract}
A form of microcirculation called vasculogenic mimicry (VM), which constitutes a novel approach for tumor blood supply in certain highly aggressive malignant tumors, was recently reported to contribute to tumor metastasis and poor prognosis in melanoma patients. Development of strategies to target tumor VM may be significant to reduce the recurrence and metastasis of melanoma. Norcantharidin (NCTD) has been shown to inhibit tumor growth and VM of human gallbladder carcinomas. Besides, NCTD could induce melanoma cell apoptosis. However, whether NCTD can inhibit the growth and VM formation of melanoma has not been evaluated. The present study aims to investigate the anti-VM activity of NCTD as a VM inhibitor for melanoma and its potential mechanisms. The anti-VM activity of NCTD was determined in human melanoma A375 cells and xenografts in vitro and in vivo. The findings indicate that NCTD inhibits tumor growth and VM formation of melanoma both in vitro and in vivo by suppressing matrix metalloproteinase-2 expression. The results suggest that NCTD is a potential therapeutic agent targeting VM in melanoma.
\end{abstract}

\section{Introduction}

Melanoma is the least common but most serious form of skin cancer (1). Rich blood supply affects the growth and metastasis of melanoma (2). A unique form of microcirculation called vasculogenic mimicry (VM), which is composed strictly of tumor cells without endothelial cells and efficiently supplies blood to tumor cells, has been reported (3). VM is associated with poor prognosis for patients with certain

Correspondence to: Mrs. Shan Yan, Institute of Molecular and Clinical Medicine, Kunming Medical University, 1168 Chun-Rong Western Road, Yu Hua Street, Cheng-Gong New City, Yunnan, Kunming 650500, P.R. China

E-mail: shanyan201412@126.com

Key words: norcantharidin, vasculogenic mimicry, melanoma, matrix metalloproteinases aggressive malignant tumors, including melanoma (4), hepatocellular carcinoma (5) and breast cancer (6). It was reported that the expression and secretion of matrix metalloproteinase (MMP)-2 is important in VM formation (7). The sole application of angiogenic inhibitors proved to be ineffective on VM due to the different molecular mechanisms that exist in endothelium-dependent angiogenesis and VM $(8,9)$. Thus, it is important to develop new angiogenic inhibitors that target tumor VM or to combine anti-VM drugs with conventional chemotherapies. Traditional Chinese medicines are reported to have multifunctional antitumor activities (10).

Norcantharidin (NCTD), a demethylated analog of cantharidin, is a 7-oxabicyclo heptane-2,3-dicarboxylic acid derivative isolated from natural blister beetles that has antitumor properties in a variety of tumors such as primary hepatocellular (11) and bladder cancer (12), and the mechanism of NCTD against bladder cancer may be attributable to its anti-VM activity. NCTD also induces cell apoptosis in melanoma in vitro (13). However, whether NCTD can inhibit the tumor growth of melanoma in vivo and its underlying mechanisms remain unclear.

The current study aims to investigate the antitumor activity of NCTD as a VM inhibitor for human melanoma and its underlying mechanisms. The results indicate that NCTD inhibits tumor growth and VM of human melanoma by suppressing MMP-2 expression in vitro and in vivo. Thus, the present study reveals that NCTD may be a potential anti-VM agent for human melanoma.

\section{Materials and methods}

Cell culture. The A375 human melanoma cell line was obtained from the Cell Resource Center (Beijing, China) and was maintained in RPMI 1640 medium (Beijing Neuronbc Laboratories, Co. Ltd., Beijing, China) supplemented with $10 \%$ fetal bovine serum (FBS; HyClone; GE Healthcare Life Sciences, Logan, UT, USA) in an incubator with high-efficiency particulate arrestance class 100 filter $\left(\right.$ Forma $^{\mathrm{TM}}$ Series II; Thermo Fisher Scientific, Inc., Waltham, MA, USA) at $37^{\circ} \mathrm{C}$ with $5 \% \mathrm{CO}_{2}$.

Invasion assays. Transwell membranes ( $8-\mu \mathrm{m}$ pore size; Costar; Corning, Life Sciences, Cambridge, UK) were coated with Matrigel (1 mg/ml; BD Biosciences, Franklin Lakes, NJ, USA) 
prior to cell passage. A375 cells $\left(1 \times 10^{5}\right)$ were seeded into the upper wells in RPMI 1640 medium supplemented with $0.2 \%$ FBS. Cells were untreated (control group) or treated with $28 \mu \mathrm{g} / \mathrm{ml}(1 / 2$ half maximal inhibitory concentration) NCTD (NCTD group). NCTD was purchased from Jiangsu Kangxi Pharmaceutical Works (Jiangsu, China) in fresh culture medium. Lower wells contained RPMI 1640 medium supplemented with $20 \%$ FBS. After $24 \mathrm{~h}$ of incubation at $37^{\circ} \mathrm{C}$, non-invading cells were removed from the upper surface of the membrane, and the cells that invaded each membrane were stained with a crystal violet solution and counted as described previously (7).

Three-dimensional (3-D) cultures. Matrigel was thawed at $4^{\circ} \mathrm{C}$, and $20 \mu \mathrm{l}$ was quickly added to each well of a 96-well plate and allowed to solidify for $40 \mathrm{~min}$ at $37^{\circ} \mathrm{C}$ in a humidified $5 \% \mathrm{CO}_{2}$ incubator. Tumor cells were seeded in complete RPMI 1640 medium onto the plate and incubated with or without NCTD $(28 \mu \mathrm{g} / \mathrm{ml})$ at $37^{\circ} \mathrm{C}$ for 12 and $24 \mathrm{~h}$, respectively. Vasculogenic-like structure formation was filmed under an inverted phase-contrast light microscope.

Tumor xenografts. Mice (4-6 week-old BALB/c nu/nu male mice; Shanghai Laboratory Animal Center, Shanghai, China) weighing 18-24 g were randomly divided into the control group and the NCTD group ( $n=10 /$ group). The mice were housed according to the official recommendations of the Chinese Community Guidelines (14). Tumors were established by inoculation of $1 \times 10^{6} \mathrm{~A} 375$ cells suspended in $100 \mu \mathrm{l}$ normal saline into the right back of the mice by subcutaneous injection. After the tumors had grown to a size of $\sim 100 \mathrm{~mm}^{3}$, NCTD was administered by intraperitoneal injection at $28 \mathrm{mg} / \mathrm{kg}$ (1/5 half maximal lethal dose) in $0.1 \mathrm{ml}$ normal saline for 13 consecutive days. Control animals were administered $0.1 \mathrm{ml}$ normal saline as a vehicle control. The maximum diameter (a) and the minimum diameter (b) were measured with calipers every 2 days. The tumor volume (V) was calculated by the following formula: $\mathrm{V}=a b^{2} \times 0.5$. The tumor weight was also evaluated once the mice were sacrificed. All animal procedures were performed according to an approved protocol by the Animal Ethical Committee of the Tianjin International Joint Academy of Biotechnology and Medicine (Tianjin, China).

Immunohistochemical analysis. Xenografted tumors from the mice were excised, fixed in $4 \%$ paraformaldehyde for $24 \mathrm{~h}$, and then embedded in paraffin for histological studies. Paraffin-embedded tissues were sectioned into slices of $4-\mu \mathrm{m}$ thickness for histological studies. Dewaxed and rehydrated tissue sections were subjected to antigen retrieval processes. Upon blocking, the sections were incubated overnight at $4^{\circ} \mathrm{C}$ with anti-cluster of differentiation (CD) 31 antibody (dilution 1:50; catalogue number JC70; Neomarkers, Fremont, CA, USA) or anti-MMP-2 antibody (dilution 1:200; catalogue number ab37150; Abcam, Cambridge, UK). Negative controls were prepared using PBS instead of the primary antibodies. Upon washing with PBS, the sections were incubated with a goat anti-mouse EnVision kit (Genentech, South San Francisco, CA, USA) for $40 \mathrm{~min}$ at $37^{\circ} \mathrm{C}$, and then incubated with 3,3'-diaminobenzidine chromogen for $10 \mathrm{~min}$. The slides for CD31-periodic acid-Schiff (PAS; Beijing Zhongshan Jinqiao Biotechnology Co. Ltd., Beijing, China) double staining were
Table I. Concentration and activity state of MMP-2 in serum-free conditioned medium from melanoma cells cultured on Matrigel.

\begin{tabular}{lcc}
\hline $\begin{array}{l}\text { Serum-free } \\
\text { conditioned medium }\end{array}$ & $\begin{array}{c}\text { MMP-2 } \\
(\mathrm{ng} / \mathrm{ml})\end{array}$ & $\begin{array}{c}\text { MMP-2 } \\
\text { activity }(\mathrm{ng} / \mathrm{ml})\end{array}$ \\
\hline Control & $367.82 \pm 14.8$ & $28.09 \pm 5.64$ \\
NCTD & $43.15 \pm 5.67^{\mathrm{a}}$ & $2.29 \pm 0.18^{\mathrm{a}}$ \\
\hline
\end{tabular}

${ }^{\mathrm{a}} \mathrm{P}<0.05$ compared with the control group. NCTD, norcantharidin; MMP, matrix metalloproteinase.

then exposed to a $0.5 \%$ periodic acid solution for $15 \mathrm{~min}$ and subsequently incubated in Schiff solution for $20 \mathrm{~min}$ in a dark chamber. Subsequently, the slides were washed with distilled water for $3 \mathrm{~min}$ and counterstained with hematoxylin. Multiplication of intensity and percentage scores was utilized to determine the staining index result.

Zymography assays and MMP-2 protein concentration determination. Gelatin zymography was used to examine the levels of MMP-2 activity in A375 cells that were either untreated (control group) or treated with $28 \mu \mathrm{g} / \mathrm{ml} \mathrm{NTCD}$ for $48 \mathrm{~h}$. The culture media were collected and subjected to $10 \%$ SDS-PAGE using $0.01 \% \mathrm{w} / \mathrm{v}$ gelatin. The gel was washed twice in $2.5 \%$ (w/v) Triton X-100 solution and incubated overnight at $37^{\circ} \mathrm{C}$ in developing buffer [50 mmol/1 Tris/ $\mathrm{HCl}(\mathrm{pH} 7.4), 10 \mathrm{mmol} / 1$ $\mathrm{CaCl}_{2}, 5 \mathrm{mmol} / 1 \mathrm{ZnCl}_{2}$ and $0.05 \% \mathrm{Brij}^{\mathrm{TM}} 35$ Surfact-Amps ${ }^{\mathrm{TM}}$ Detergent Solution (Thermo Fisher Scientific, Inc.)]. The gels were subsequently stained with Coomassie Brilliant Blue R250 and destained until the wash remained clear and cleared zones associated with MMP activity were apparent. The concentrations of exogenous pro-MMP-2 and active MMP-2 in cell culture supernatants were assessed using the MMP-2 Biotrak Activity Assay (GE Healthcare Life Sciences, Chalfont, UK) according to the manufacturer's guidelines.

Statistical analysis. All data were reported as the mean \pm standard error of the mean and evaluated using SPSS 17.0 (SPSS Inc., Chicago, IL, USA). Student-Newman-Keuls $t$ tests were used to evaluate differences between two groups. $\mathrm{P}<0.05$ was considered to indicate a statistically significant difference.

\section{Results}

NCTD inhibits the invasion of A375 cells in vitro. Matrigel-coated transwell plates were used to assess the effects of NCTD on the ability of A375 melanoma cells to invade a basement membrane matrix. The results revealed that NCTD significantly reduced the number of cells that invaded through the Matrigel matrix after $24 \mathrm{~h}$ of treatment $(\mathrm{P}=0.005)$ (Fig. 1A).

NCTD inhibits tube formation by A375 cells in vitro. The vasculogenic-like network formation ability of melanoma cells was assessed in vitro by seeding the cells onto Matrigel-coated plates and then observing the cells under an inverted phase-contrast light microscope. As shown in Fig. 1B, the formation of VM 
A
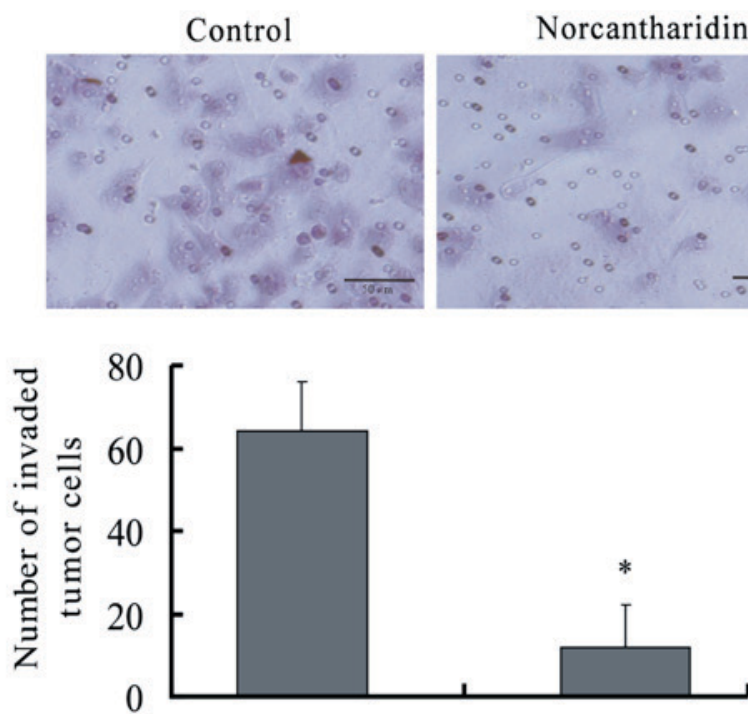

Control

Norcantharidin

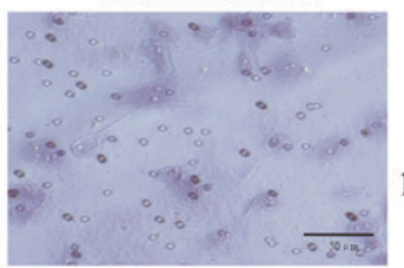

$12 \mathrm{~h}$

$24 \mathrm{~h}$
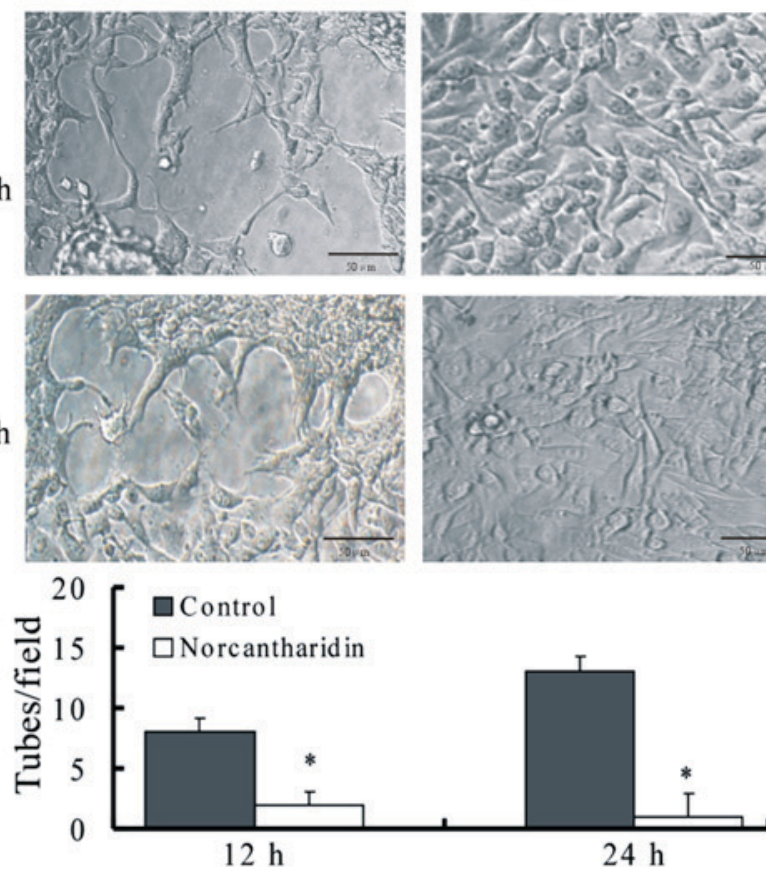

Norcantharidin
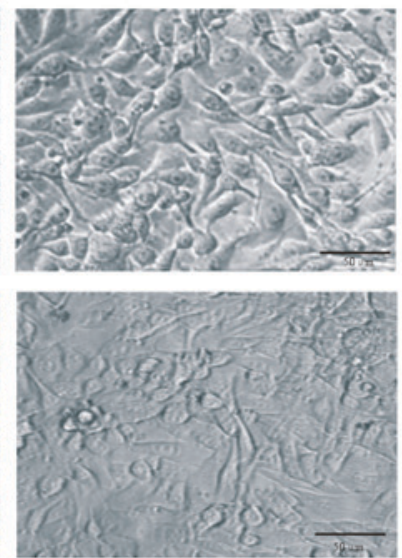

$24 \mathrm{~h}$

Figure 1. NCTD inhibits the invasion and capillary-like tube formation abilities of A375 melanoma cells in vitro. (A) Inhibitory effect of NCTD on the invasive ability of A375 melanoma cells in vitro. Upper panel, representative images of cells from the transwell invasion assay. Magnification, x200. Lower panel, quantitative analysis indicating that fewer melanoma cells invaded through the Matrigel matrix following NCTD treatment compared with those in the control group. (B) NCTD decreases the capillary-like tube formation ability of melanoma cells in vitro. Upper panel, NCTD impaired the vessel-like networks formed by A375 melanoma cells cultured on Matrigel at 12 and $24 \mathrm{~h}$. Magnification, $\mathrm{x} 400$. Lower panel, quantitative analysis indicating that less capillary-like tubes were formed in cells subjected to NCTD treatment compared with those in the control group. ${ }^{*} \mathrm{P}<0.05$ compared with the control group ( $=3$ ). NCTD, norcantharidin.

networks by A375 melanoma cells was disrupted by the addition of NCTD for 12 or $24 \mathrm{~h}$.

NCTD inhibits melanoma growth and VM formation in mice. To investigate the efficacy of NCTD in inhibiting melanoma growth, the tumor sizes of melanoma-bearing mice were measured once every 2 days throughout the experiment. At the end of the experiment, the volume and weight of the xenografts in the NCTD group decreased significantly with increased tumor inhibition in comparison with those of the control group (Fig. 2). CD31-PAS double staining was used to identify VM in the xenografts on day 21 of tumor inoculation. Microscopically, the xenografts in the control group exhibited tumor cell-lined channels containing red blood cells (Fig. 3) without any evidence of tumor necrosis. The channels consisted of tumor cells negative for CD31 and positive for PAS. By contrast, VM could hardly be observed in the tumor tissues treated with NCTD, while large areas of necrosis were easily detected, suggesting that NCTD inhibits the VM formation of melanoma xenografts in vivo.

NCTD downregulates the expression and activity of MMP-2. MMP-2 is a key player in VM formation (15). Thus, to explore the possible mechanisms of NCTD effects on tumor growth and VM of human melanoma in vitro and in vivo, in the present study, the expression and activity of MMP-2 protein from sections of melanoma xenografts and supernatants of $3-D$ culture samples were determined. The results indicated that MMP-2 expression in the in vivo xenografts of the NCTD
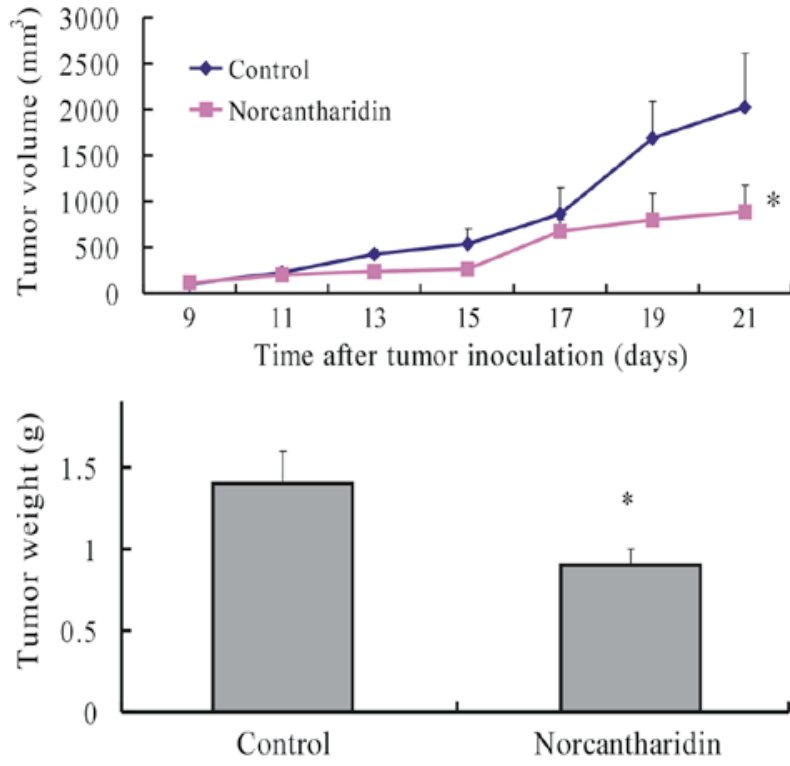

Figure 2. Norcantharidin inhibits A375 human melanoma tumor growth. Plot of tumor volume (upper panel) or tumor weight (lower panel) vs. the number of days post-tumor inoculation of melanoma-bearing mice in different groups. ${ }^{*} \mathrm{P}<0.05$ compared with the control group. Data are shown as the mean \pm standard error of the mean ( $n=10$ mice/group).

group was significantly lower than that of the control group (Fig. 4A). Furthermore, the addition of NCTD decreased both the expression and activity of MMP-2 in the 3-D culture samples (Fig. 4B and Table I). 

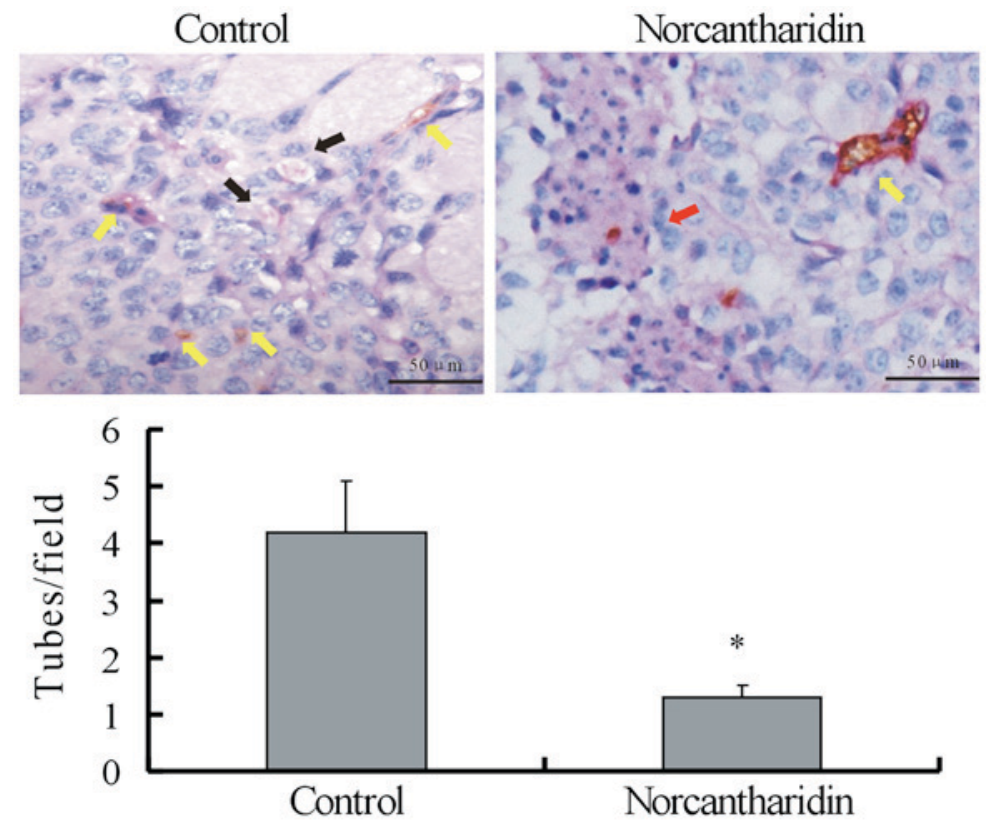

Figure 3. NCTD decreases the capillary-like tube formation ability of A375 melanoma xenografts in vivo. Upper panel, representative images of the presence of VM in tissue samples of A375 melanoma xenografts with or without treatment with NCTD. In the control group, CD31-periodic acid-Schiff staining was used for the morphological observations of VM formed by tumor cells without CD31-positively stained endothelial cells (black arrow). Red blood cells were present in the center of the channels. The yellow arrow points to CD31-positively stained endothelium-dependent vessels in the same field. In the NCTD-treated group, VM was not observed, while large areas of necrosis were easily noticed (red arrow). Yellow arrow, endothelium-dependent vessels. Magnification, x400. Lower panel, quantitative analysis indicating that less capillary-like tubes were formed upon NCTD treatment in the treated group compared with those in the control group. ${ }^{*} \mathrm{P}<0.05$ compared with the control group. NCTD, norcantharidin; $\mathrm{CD}$, cluster of differentiation; $\mathrm{VM}$, vasculogenic mimicry.

A

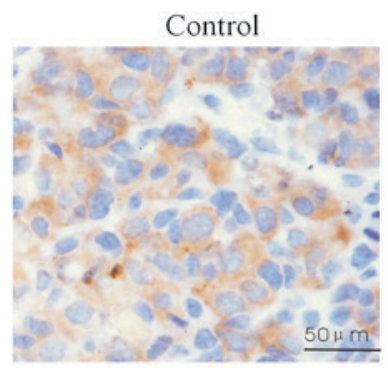

B

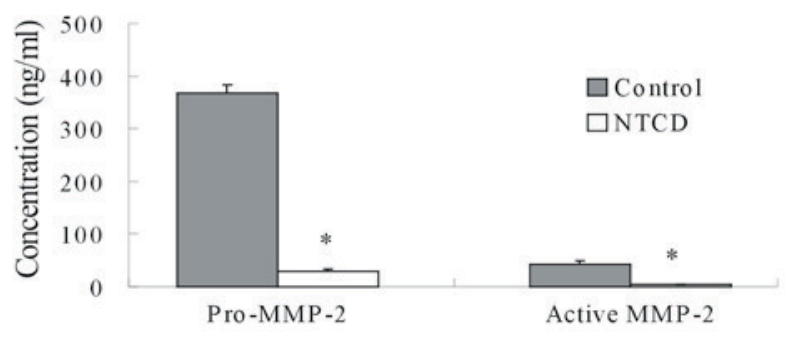

NTCD

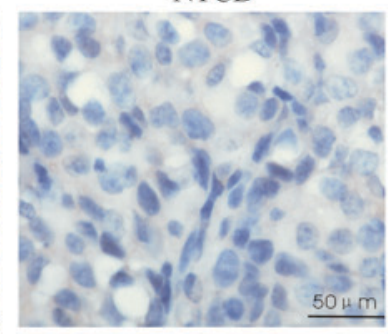

Active MMP-2
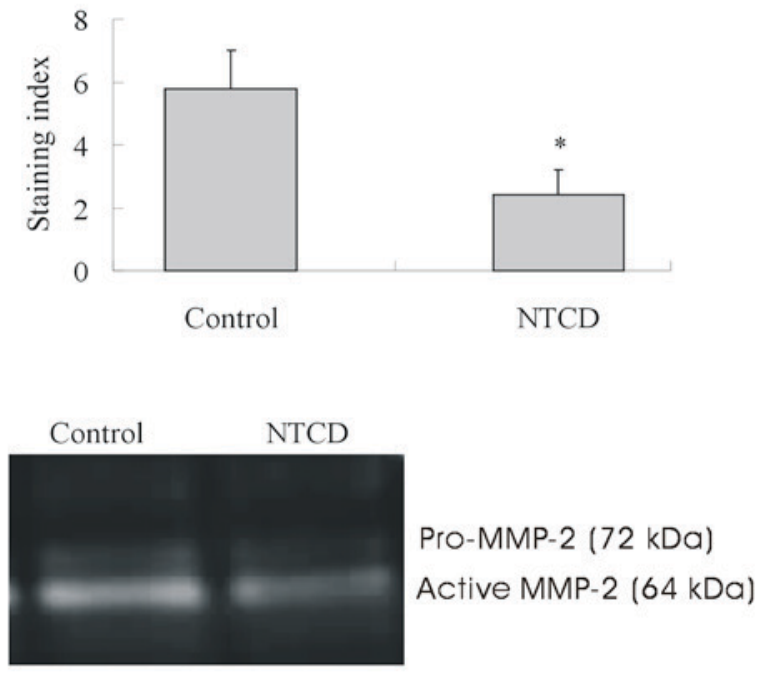

Figure 4. Expression and activity of MMP-2 protein from (A) sections of A375 melanoma xenografts and (B) serum-free conditioned medium from melanoma cells cultured on Matrigel. (A) Upper panel, representative images of MMP-2 expression in A375 melanoma xenografts. Positive staining of MMP-2 could be observed in the cytoplasm of tumor cells. The lower panel indicates the quantitative analysis of MMP-2 expression, and lower expression was observed in the NCTD group compared with that in the control group ("P<0.05). (B) The concentration and activity of MMP-2 protein from supernatants of three-dimensional culture samples were measured either by MMP-2 Biotrak Activity Assay (left) or by zymography assay (right). Both the expression and activity of MMP-2 protein in the NCTD group were significantly lower than those in the control group ("P<0.05). NCTD, norcantharidin; MMP, matrix metalloproteinase.

\section{Discussion}

The present study demonstrates that NCTD inhibits tumor growth and VM of melanoma by suppressing MMP-2 expression.

Widespread metastasis caused by increased cell motility and a rich blood supply of tumor cells is the main cause of the poor prognosis of melanoma patients (16). Traditional anti-angiogenic drugs, including bevacizumab, sunitinib, angiostatin and endostatin, have yielded disappointing results on the management of melanoma, since VM exists as a particular microcirculation pattern, and the sole blockage of angiogenesis may not be effective $(9,17-20)$. 
Thus, the development of anti-VM drugs for the treatment of melanoma with VM is an urgent concern. NCTD is a demethylated and low-cytotoxic derivative of cantharidin (12). It has antitumor properties, hypotoxicity in a variety of tumor and apoptosis-promoting effects in melanoma in vitro (13). NCTD also inhibits VM formation in human gallbladder carcinomas (21). The present study further investigated the anti-VM activity of NCTD as a VM inhibitor for human melanoma. The results indicate that NCTD inhibits the growth and VM formation of melanoma both in vitro and in vivo, thus suggesting that NCTD may be a potential therapeutic agent targeting VM in melanoma.

We also sought to determine the possible mechanism of the inhibitory effects of NCTD on the growth and VM formation of melanoma. The MMP-2 protein is considered to play a key role in VM formation in melanoma via the cleavage of the laminin (Ln)-5 $\gamma 2$ chain into two segments (Ln-5 $\gamma 2 \mathrm{x}$ and Ln-5 $\gamma 2^{\prime}$ ), which results in VM formation (15). Zhang et al reported that NCTD inhibits tumor growth and VM of human gallbladder carcinomas by suppressing the phosphatidylinositol-4,5-bisphosphate 3-kinase (PI3-K)/MMP-2/Ln-5 2 signaling pathway (12). Thus, in the current study, the effects of NCTD on MMP-2 protein expression and activity in melanoma were determined both in vivo and in vitro. The results demonstrate that NCTD not only inhibits VM formation of melanoma cells and xenografts, but also downregulates MMP-2 expression in vitro and in vivo. These results suggest that the PI3-K/MMP-2/Ln-5 $\gamma 2$ signaling pathway may also be the underlying molecular mechanism of the inhibitory effects of NCTD on the growth and VM formation of melanoma. Therefore, NCTD could be used as a potential anti-VM inhibitor in melanoma treatment.

In conclusion, the present study demonstrated that NCTD inhibits the growth and VM formation of melanoma by suppressing MMP-2 expression. NCTD may be used as a potential therapeutic agent targeting VM in melanoma. Further investigations are necessary to verify other molecular mechanisms of the inhibitory effects of NCTD on the VM formation of melanoma.

\section{References}

1. Jemal A, Siegel R, Xu J and Ward E: Cancer statistics, 2010. CA Cancer J Clin 60: 277-300, 2010.

2. Neitzel LT, Neitzel CD, Magee KL and Malafa MP: Angiogenesis correlates with metastasis in melanoma. Ann Surg Oncol 6 : 70-74, 1999.

3. Maniotis AJ, Folberg R, Hess A, Seftor EA, Gardner LM, Pe'er J, Trent JM, Meltzer PS and Hendrix MJ: Vascular channel formation by human melanoma cells in vivo and in vitro: Vasculogenic mimicry. Am J Pathol 155: 739-752, 1999.
4. Warso MA, Maniotis AJ, Chen X, Majumdar D, Patel MK, Shilkaitis A, Gupta TK and Folberg R: Prognostic significance of periodic acid-Schiff-positive patterns in primary cutaneous melanoma. Clin Cancer Res 7: 473-477, 2001.

5. Liu WB, Xu GL, Jia WD, Li JS, Ma JL, Chen K, Wang ZH, Ge YS, Ren WH, Yu JH, et al: Prognostic significance and mechanisms of patterned matrix vasculogenic mimicry in hepatocellular carcinoma. Med Oncol 28 (Suppl 1): S228-S238, 2011.

6. Shirakawa K, Wakasugi H, Heike Y, Watanabe I, Yamada S, Saito Kand Konishi F: Vasculogenic mimicry and pseudo-comedo formation in breast cancer. Int J Cancer 99: 821-828, 2002.

7. Seftor EA, Meltzer PS, Kirschmann DA, Pe'er J, Maniotis AJ, Trent JM, Folberg R and Hendrix MJ: Molecular determinants of human uveal melanoma invasion and metastasis. Clin Exp Metastasis 19: 233-246, 2002.

8. van der Schaft DW, Seftor RE, Seftor EA, Hess AR, Gruman LM, Kirschmann DA, Yokoyama Y, Griffioen AW and Hendrix MJ: Effects of angiogenesis inhibitors on vascular network formation by human endothelial and melanoma cells. J Natl Cancer Inst 96: 1473-1477, 2004.

9. Rice $\mathrm{C}$ and Huang LE: From antiangiogenesis to hypoxia: Current research and future directions. Cancer Manag Res 3: 9-16, 2010.

10. Sun M, Han J, Duan J, Cui Y, Wang T, Zhang W, Liu W, Hong J, Yao M, Xiong S and Yan X: Novel antitumor activities of Kushen flavonoids in vitro and in vivo. Phytother Res 21: 269-277, 2007.

11. Yeh CB, Hsieh MJ, Hsieh YH, Chien MH, Chiou HL and Yang SF: Antimetastatic effects of norcantharidin on hepatocellular carcinoma by transcriptional inhibition of MMP-9 through modulation of NF-kB activity. PLoS One 7: e31055, 2012.

12. Zhang JT, Sun W, Zhang WZ, Ge CY, Liu ZY, Zhao ZM, Lu XS and Fan YZ: Norcantharidin inhibits tumor growth and vasculogenic mimicry of human gallbladder carcinomas by suppression of the PI3-K/MMPs/Ln-5 $\gamma 2$ signaling pathway. BMC Cancer 14: 193, 2014

13. An WW, Wang MW, Tashiro S, Onodera S and Ikejima T: Norcantharidin induces human melanoma A375-S2 cell apoptosis through mitochondrial and caspase pathways. J Korean Med Sci 19: 560-566, 2004

14. Qin Y,Zhang Q,Lee S,Zhong WL, Liu YR, Liu HJ,Zhao D, Chen S, Xiao T, Meng J, et al: Doxycycline reverses epithelial-to-mesenchymal transition and suppresses the proliferation and metastasis of lung cancer cells. Oncotarget 6: 40667-40679, 2015.

15. Hess AR, Seftor EA, Seftor RE and Hendrix MJ: Phosphoinositide 3-kinase regulates membrane Type 1-matrix metalloproteinase (MMP) and MMP-2 activity during melanoma cell vasculogenic mimicry. Cancer Res 63: 4757-4762, 2003.

16. Hofmann-Wellenhof R, Woltsche-Kahr I, Smolle J and Kerl H: Clinical and histological features of poor prognosis in cutaneous metastatic melanomas. J Cutan Pathol 23: 199-204, 1996.

17. Chen HX and Cleck JN: Adverse effects of anticancer agents that target the VEGF pathway. Nat Rev Clin Oncol 6: 465-477, 2009.

18. Higa GM and Abraham J: Biological mechanisms of bevacizumab-associated adverse events. Expert Rev Anticancer Ther 9: 999-1007, 2009.

19. Gille J: Antiangiogenic cancer therapies get their act together: Current developments and future prospects of growth factor- and growth factor receptor-targeted approaches. Exp Dermatol 15: 175-186, 2006.

20. Grothey A and Galanis E: Targeting angiogenesis: Progress with anti-VEGF treatment with large molecules. Nat Rev Clin Oncol 6: 507-518, 2009.

21. Zhang JT, Fan YZ, Chen CQ, Zhao ZM and Sun W: Norcantharidin: A potential antiangiogenic agent for gallbladder cancers in vitro and in vivo. Int J Oncol 40: 1501-1514, 2012 